\title{
Daiobotanpito combined with antibiotic therapy for treatment of acute diverticulitis: a study protocol for a randomized controlled trial
}

Keiko Ogawa-Ochiai ( $\nabla$ ikkandoo@gmail.com )

Kanazawa Daigaku https://orcid.org/0000-0002-3277-0646

Kenichi Yoshimura

Kanazawa Daigaku

Akiko Shirai

Kanazawa Daigaku

Seisho Sakai

Kanazawa Daigaku

Hideki Moriyama

Kanazawa Daigaku

Keishi Nakamura

Kanazawa Daigaku

Toshinori Murayama

Kanazawa Daigaku

Hideki Ishikawa

Kyoto Prefectural University of Medicine

Study protocol

Keywords: Japanese traditional (Kampo) medicine, daiobotanpito, diverticulitis, da huang mu dan tang, randomized controlled trial

Posted Date: May 7th, 2019

DOI: https://doi.org/10.21203/rs.2.9452/v1

License: (c) (i) This work is licensed under a Creative Commons Attribution 4.0 International License.

Read Full License 


\section{Abstract}

Background: Colonic diverticular disease has been increasing in prevalence due to the rapidly aging global population, but standard treatment has not changed dramatically in recent years. Daiobotanpito (DBT; Da Huang Mu Dan Tang in Chinese) has been used in medical treatment of acute abdominal abscesses, such as appendicitis or diverticulitis in traditional Japanese (Kampo) medicine for many years, based on more than 3000 years of experience. Prior to this study, a retrospective open-label trial was conducted to compare patients with acute diverticulitis who received oral DBT combined with intravenous antibiotics with those who received intravenous antibiotic alone; it showed a positive effect of DBT on acute diverticulitis. We aim to investigate whether moderate to severe acute diverticulitis shows greater improvement with intravenous antibiotics plus orally administered DBT compared with intravenous antibiotics with placebo. Methods: This is a two-group, randomized, double-blind, placebocontrolled, multi-center trial, which is designed to evaluate the efficacy and safety of DBT for moderate to severe diverticulitis patients treated with intravenous antibiotics. Eligible participants will be randomized to either a treatment group receiving a 10-day oral DBT regimen plus conventional therapy or a control group receiving a 10-day placebo regimen plus conventional therapy. The primary outcome will be the rate of fever reduction within 3 days or/and abdominal pain relief within 4 days. Secondary endpoints will include hospitalization days, changes in inflammatory response (CRP, WBC, neutrophil count), thermal type, number of days before beginning food intake, recurrence rate (observation for 1 year after registration), and adverse event expression rate. Assessments will be performed at baseline and on the day of discharge. The recurrence rate will be recorded at 1 year after registration. Discussion: This study is expected to provide evidence to support the clinical benefits of DBT in the treatment of acute diverticulitis. It may also provide evidence regarding the efficacy and safety of DBT in the recurrence of acute diverticulitis. Trial registration: UMIN000027381, registered on April 27th, 2017; https://upload.umin.ac.jp/cgi-bin/ctr/ctr_view_reg.cgi?recptno=R000031377.

\section{Background}

The prevalence of diverticular disease is estimated to range between $20 \%$ and $60 \%$ in the general population worldwide [1, 2]. Although $75 \%$ of patients with diverticulosis remain symptom-free during their lifetimes, the prevalence of patients who require medical or surgical treatment has increased by $16 \%$ in the last 20 years, thereby increasing morbidity associated with this condition [3-5]. According to the Recommendations from the American Gastroenterological Association on treatment and diagnosis [6] and the Guidelines for Colonic Diverticulitis of the Japan Gastroenterological Association [7], the initial treatment of uncomplicated colonic acute diverticulitis is bowel rest (i.e., fasting) and broad-spectrum antibiotics. Notably, despite the presence of abscess or perforation with colonic diverticulitis, conservative treatment is typically performed for limited peritonitis and abscess. Patients who do not respond to medical therapy should be considered for surgery depending on the clinical situation. Elective resection is recommended after two well-documented attacks, depending on the patient's age and medical fitness, as well as the severity of the attack. 
Moreover, colonic diverticular disease has been increasing in prevalence due to the rapidly aging global population [6]. However, standard treatment has not changed noticeably in recent years.

The unique application of traditional Japanese (Kampo) medicine is gradually attracting worldwide attention. In Kampo medicine, daiobotanpito (DBT; Da-Huang-Mu-Dan-Tang) is used for patients with Yang-excess in the interior layers of the body, who exhibit signs of local Qi-congestion and blood stagnation. It has traditionally been used for abscesses of the intestine, such as diverticulitis or appendicitis. From the perspective of Kampo medicine, DBT drains heat, dispels blood stasis, disperses clumping, and reduces swelling. It is primarily used in patients with abdominal distention and constipation. Daiobotanpito consists of five crude drugs: Rhei Rhizoma, Natrium sulfuricum, Moutan Cortex, Persicae Semen, and Benincaseae Semen. Notably, Rhei Radix et Rhizoma and Moutan Cortex drain heat and dispel blood stasis. These aspects are attributed to heat and constraints that allow the formation of toxins, which secondarily leads to the formation of pus. The appropriate strategy of Kampo medicine is to discharge the toxin, eliminate the phlegm, clear the heat, and open the restraint [8].

As described above, surgery should be performed in patients who do not respond to medical therapy in difficult or complicated clinical situations. DBT may be an alternative or supportive therapy for patients with acute diverticulitis, which may enable these patients to avoid surgical treatment. Prior to this study, a retrospective open-label trial was conducted to compare patients with acute diverticulitis who received oral DBT combined with intravenous antibiotics with those who received intravenous antibiotics alone; importantly, it showed a positive effect of DBT for treatment of acute diverticulitis. Thirty-four patients were included: 11 patients in group 1 with DBT, and 23 patients in group 2 without DBT. There was a significantly improved outcome in the group treated with DBT, compared to the group without DBT, when comparing duration of fever, abdominal pain, and administration of antibiotics [9].

The authors hypothesize that combined treatment with oral DBT is superior to the standard therapy for acute diverticulitis. This study aims to investigate the effectiveness of the treatment of acute colonic diverticulitis with Kampo medicine, DBT.

\section{Methods/design}

\section{Study design and settings}

A brief flowchart of the entire study is shown in Fig. 1. This study is a two-group, randomized, doubleblind, placebo-controlled, multi-center trial, which will evaluate the efficacy and safety of DBT for acute diverticulitis patients. The study will be conducted throughout Japan. Patients will be recruited from the gastroenterology inpatient departments of 13 hospitals. Informed consent will be obtained from all study participants. The study protocol was designed in accordance with the ethical principles in the Declaration of Helsinki and regional regulations. Central ethical approval of this study has been confirmed from the Central Review Board of Kanazawa University Hospital (ref approval no.6058) and we will not begin recruiting at other centres in the trial until local ethical approval has been obtained. This study is registered at http://www.umin.ac.jp/ctr/index-j.htm (UMIN000027381). The protocol includes elements 
recommended in the Standard Protocol Items: Recommendations for Interventional Trials (SPIRIT) [10] checklist (Additional file 1).

\section{Setting and participants}

This study will be conducted in Japan. Patients will be recruited from the gastroenterology and surgery inpatient department at each of the hospitals in different districts in Japan.

Participants who meet the following inclusion criteria are eligible:

1. Moderate to severe diverticulitis: diagnosis will be based on computed tomography (CT) images showing diverticula-like structure combined with tenderness or abdominal pain, a thickening of colon wall, signs of inflammation of pericolonic fat, tissue density, and vascular involvement. Pericolonic abscess, free air or extravasation, and accumulation of fluid will also be assessed to predict prognosis.

2. Age of at least 20 years and less than 75 years

3. Ability to communicate with the investigators

4. Abdominal pain and/or fever $>37.5^{\circ} \mathrm{C}$

5. Provision of written informed consent for participation after receiving sufficient explanation for participation in this study and achieving sufficient understanding.

Participants who meet the following exclusion criteria will not be enrolled:

1. Severe dysfunction in the following organs, based on blood tests within 4 weeks before treatment starts:

a. Renal function: serum creatinine value 1.5 -fold greater than the upper limit of the institutional standard

b. Liver function: serum AST value or serum ALT value 3-fold greater than the upper limit of the institutional standard

c. Central nervous function: encephalopathy or a patient suspected of it

d. Electrolytes: serum sodium $<125 \mathrm{mEq} / \mathrm{L}$, serum potassium $<3.3 \mathrm{mEq} / \mathrm{L}$

2. Highly likely to exhibit abscess perforation due to malnutrition (Alb $<2.5 \mathrm{~g} / \mathrm{dL}$ )

3. Symptoms of obstructive ileus

4. Chronic anorexia, abdominal pain, and/or diarrhea symptoms before the onset of colorectal diverticulitis 
5. Performance status (an indicator of general condition and the degree of restriction of the patient's daily life) of $\geq 3$, before the onset of colorectal diverticulitis

6. History of DBT administration

7. Treatment with insulin preparations

8. Immunocompromised status

9. Pregnancy or postpartum status

10. Advanced allergy to Kampo formulas

11. Doctor's judgement to be inappropriate for inclusion in this study.

Randomization, allocation concealment, and blinding

The web-based online randomization system used in this trial was provided by an independent academic research organization at Kyoto Prefectural University of Medicine. Enrolled patients who provide informed consent will be randomized 1:1 in a blinded manner into either an experimental treatment group receiving a 10-day DBT regimen plus conventional therapy, or a control group receiving a 10-day placebo regimen plus conventional therapy. All participants and investigators will be blinded to treatment allocation.

\section{Interventions}

We will use DBT extract, which consists of five crude drugs in fixed proportions: Rhei Rhizoma $(2.0 \mathrm{~g})$, Natrium sulfuricum (1.8 g), Moutan Cortex (4.0 g), Persicae Semen (4.0 g), and Benincasae Semen (6.0 g), in $7.5 \mathrm{~g}$ of extract. DBT extract (Tsumura, Tokyo)-or placebo manufactured by Tsumura Co., Ltd., based on good manufacturing practice standards-at $2.5 \mathrm{~g}$ will be administered three times per day, $7.5 \mathrm{~g}$ per day, for 10 days. The administration of DBT or placebo begins immediately after informed consent and web-based randomization. No restrictions will be imposed on the standard treatments for acute diverticulitis or any other disease while DBT is administered. If the clinical evolution is appropriate, a regular diet will be initiated, and the patient will be discharged; the patient will return as an outpatient 1 week later. We will ask patients whether they have relapsed, 1 year after discharge. When any serious adverse event occurs or patients do not wish to further participate in the trial, they will be excluded from the examination.

\section{Data collection}

The study data collection process is outlined in Table 1.

[Table 1 near here]

Primary Endpoint 
Treatment success rate

If criteria below are satisfied, treatment will be defined as successful.

1. In a patient with a fever of $37.5^{\circ} \mathrm{C}$ and abdominal pain requiring treatment at baseline, both fever reduction and disappearance of abdominal pain are confirmed.

2. In a patient with abdominal pain requiring treatment, without fever of $\geq 37.5^{\circ} \mathrm{C}$ at baseline, disappearance of abdominal pain is observed.

For patients who use any antipyretic analgesic after randomization, the disappearance of abdominal pain is confirmed if no abdominal pain is present at $\geq 6$ hours from the administration of antipyretic analgesic.

\section{Secondary Endpoints}

1. Number of hospital days: Number of days until discharge, including the registration date, after trial registration.

2. Change in inflammatory response (CRP, WBC, neutrophil count): Differences between the value at the time of registration (baseline value) of each measurement and the value at each measurement day.

3. Thermal type: Differences between the values at the time of registration (baseline value) of body temperature and the values on each measurement day.

4. Number of days until oral ingestion: Number of days until the beginning of oral intake, including the registration date, after trial registration.

5. Recurrence rate (1-year follow-up observation): Rate of recurrence at 1 year after trial registration. After discharge, recurrence is regarded as diagnosis of colonic diverticulitis.

6. Adverse events (types and frequency of side effects): Side effects will be recorded for each observed adverse event, and the type and frequency will be determined.

Statistical consideration

Sample size

Calculation of the sample size in this trial was based on treatment success rate. Based on the results of our retrospective study, we expected that the treatment success rate would be $75 \%$ in the experimental treatment group and $50 \%$ in the control group. Based on this expectation, a sample size of 150 patients (75 per arm) would provide the trial with $90 \%$ power to detect the superiority of experimental treatment over control, at a two-sided alpha level of $5 \%$. 
Treatment success rate will be compared between groups using the Pearson chi-square test. Time to confirmed treatment-success will be summarized using the Kaplan-Meier method. Continuous variables will be summarized as mean and standard deviations, and comparisons will be made using the unpaired Student's t-test. Categorical variables will be summarized as frequency and proportions, and comparisons will be made using the chi-square test. Two-sided p-values less than 0.05 will be considered statistically significant.

\section{Quality control and trial management}

The management structure comprises the principle investigator (PI), a trial management group, and a data monitoring committee. The trial management group is responsible for conducting the trial and will meet monthly to discuss trial progress. The PI will visit each collaborative hospital for face-to-face meetings and sharing of information to promote patient recruitment. The data monitoring committee will review safety and efficacy data. All data will be monitored every month by central monitoring method. Additional monitoring may be performed at the discretion of the monitoring manager. The data monitoring committee met once, prior to the start of patient recruitment. At least twice per year, participating investigators, research assistants, and research nurses will be required to attend a training workshop for clinical research to ensure strict adherence to the study protocol and familiarity with the trial administration process. The data collected in this trial will comprise information recorded in case report forms and questionnaires. Data quality will be checked regularly by research assistants and overseen by monitors; all modifications will be marked on case report forms, and data managers will recheck the data before they are officially logged. The database will be locked after all data have been cleaned. If participants withdraw from the trial during the study period, the reasons will be documented, and the dropout rate will be statistically analyzed.

\section{Discussion}

The main purpose of this study is to assess the treatment effect of DBT in moderate to severe acute diverticulitis. The secondary aim of this study is to investigate the preventive effect of DBT on colonic diverticulitis. Patients with a history of diverticulitis are advised to consume a high-fiber diet or ingest fiber supplements, minimize red meat consumption, avoid non-aspirin non-steroidal anti-inflammatory drugs, stop smoking, maintain or achieve a healthy body weight, and exercise regularly [6]. Although multiple studies have investigated the methods of colonic diverticulitis prevention, the existing results are not supported by high-quality evidence. In this study, we will investigate differences between treatment with DBT combined with antibiotics, relative to treatment with placebo.

Diverticula can occur at any site in the colon. However, they occur primarily in the sigmoid colon in Western countries; in contrast, the right side or bilateral colon is more commonly involved in Japan. The standard treatment for acute uncomplicated diverticulitis has been bowel rest (i.e., fasting), intravenous fluids, and intravenous antibiotics. There will be a diverse assortment of antibiotics used for patients in this study, based on variability in the antibiotics used in clinical practice among centers in the study. 
Additionally, while many trials have focused on surgical treatment of acute diverticulitis, there have been few studies dedicated to medical treatment of this condition. A review of the published data revealed that there is no standardization regarding the medical treatment of uncomplicated acute diverticulitis [11]. In this study, we will not limit the types of antibiotics; all antibiotics will be noted in the case report forms.

In Kampo medicine, internal abscesses generally comprise abscesses of the lungs and intestines. Similar to external abscesses, they are attributed to heat and constraint, which allow the formation of toxins and secondarily lead to the formation of pus. The appropriate strategy is to discharge the toxin, eliminate the phlegm, clear the heat, and open the constraint. If clumping of heat with stasis and stagnation is also present, herbs such as Rhei Radix et Rhizoma (da hungu) and Moutan Cortex (mu dan piाu) can be added to drain heat and dispel stasis [8]. In this context, DBT is one of the most suitable formulas for treatment of acute diverticulitis. DBT might have preventive effects on the recurrence of diverticulitis, although the prognosis of patients was not reported in a prior study [9].

Most herbal medicine is orally administered, and many components of these medicines come into contact with the intestinal microflora in the alimentary tract. Some are modified by intestinal bacteria before their absorption into the bloodstream from the gastrointestinal tract [12]. Although administration of intravenous antibiotics may influence intestinal microflora and the effect of DBT might be altered, compared with classical therapy, our previous study showed that both intravenous antibiotics and DBT could be used at the same time [9].

DBT consists of five crude drugs: Rhei Rhizoma, Natrium sulfuricum, Moutan Cortex, Persicae Semen, and Benincasae Semen. Of these, Rhei Rhizoma or rhubarb is one of the most important traditional herbal medicines, widely used in Kampo and traditional Chinese medicines for thousands of years, especially as a purgative. It might prevent bowel retention, overgrowth of toxic bacteria in patients with acute diverticulitis, and enable resolution of acute inflammation.

Studies regarding other functions of rhubarb in modern medical research in both clinical and basic science settings have revealed that rhubarb has multiple effects, including defervescence and antiinflammatory actions, as well as the expulsion of a variety of harmful materials (e.g., endogenous and exogenous toxins) from the bowel and the body.

It is shown that rhubarb protects against acute lung injury induced by lipopolysaccharide, and that rhubarb administration improves respiratory function of the body. Its effect is related to the regulation of the production of nitric oxide as well as phospholipase A, and platelet-activating factor activities [14]. These effects may be related to the improvement of inflammation in diverticulitis.

Moutan Cortex, the root cortex of Paeonia suffraticosa, is an herbal medicine widely used as an analgesic, antispasmodic, and anti-inflammatory agent. Moutan Cortex reportedly inhibits the secretions of interleukin-8, a major mediator of acute neutrophil-mediated inflammation, and macrophage chemoattractant protein-1, a potent mediator of chronic macrophage-mediated inflammation in human monocytic U937 cells [15]. Recently, Moutan Cortex was shown to protect against sepsis induced by 
lipopolysaccharide/ D-galactosamine [16]. The known chemical components of Moutan Cortex include paeonol, paeonoside, paeonolide, paeoniflorin, benzoylpaeoniflorin, oxypaeoniflorin, benzoyloxypaeoniflorin, and apiopaeonoside】

Moutan Cortex inhibited the lipopolysaccharide + interferon-怄-induced expression of inducible nitric

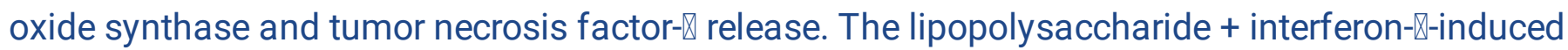
activation of nuclear factor-[B was almost entirely blocked by Moutan Cortex [16].

Persicae Semen or Prunus persica, is also well-known as a traditional medicine in Japan, China, and other Asian countries. They are frequently used as ingredients in a variety of Kampo and Chinese medicine formulas, particularly those used to treat women's diseases. The chemical constituents of the herb include cyanogenic glycosides, amygdalin and prunasin as major components, along with glycerides, sterols, and emulsion. Amygdalin is abundant in the seeds of bitter almond and apricots of the Prunus genus, and other rosaceous plants. Persicae Semen has anti-edema, anti-writhing, and anti-inflammation activities.

Each of these crude drugs has some anti-inflammatory effects, and the combination of these crude drugs may produce the synergy of Kampo medicine.

In conclusion, this study protocol may contribute to the development of an effective treatment to help relieve acute diverticulitis. It may also provide evidence on the efficacy and safety of DBT in acute diverticulitis patients.

\section{Trial Status}

Recruitment began in January 2018, and is expected to be completed in December 2019.

\section{List Of Abbreviations}

DBT: Daiobotanpito

DKT: Daiokanzoto

\section{Declarations}

\section{Ethics approval and consent to participate}

Written informed consent was obtained from all patients or their families. The study was approved by the Central Review Board of Kanazawa University Hospital, and the study was conducted in accordance with the ethical standards of the Declaration of Helsinki.

\section{Consent for publication}


Not applicable.

\section{Availability of data and material}

The data will not be available for public access because of patient privacy concerns, but will be available from the corresponding author on reasonable request.

\section{Conflict of Interest}

The study drug was received from Tsumura Co. The company has no role in study design, data collection and analysis, decision to publish, or preparation of the manuscript.

\section{Funding}

This study is supported by Japan Agency for Medical Research and Development and the Fund of Kanazawa University for Clinical Studies. The funders have no role in study design, data collection and analysis, decision to publish, or preparation of the manuscript.

\section{Authors' contributions}

$\mathrm{KO}, \mathrm{KY}, \mathrm{AS}, \mathrm{TM}$, and $\mathrm{HI}$ designed the study protocol and drafted the manuscript. SS, HM, and KN reviewed the study protocol as surgeons and drafted the manuscript. KY is responsible for the statistical design and analysis. All authors carefully read and approved the final version of the manuscript.

\section{Acknowledgements}

We thank Ms. Mie Morikawa and Ms. Aya Yoshida for their assistance.

\section{References}

[1] Parks TG. Natural history of diverticular disease of the colon. Clin Gastroenterol. 1975;4:53-69.

[2] Manousos ON, Truelove SC, Lumsden K. Prevalence of colonic diverticulosis in general population of Oxford area. Br Med J. 1967;3:762-3.

[3] Kang JY, Hoare J, Tinto A, Subramanian S, Ellis C, Majeed A, et al. Diverticular disease of the colon--on the rise: a study of hospital admissions in England between 1989/1990 and 1999/2000. Aliment Pharmacol Ther. 2003;17:1189-95.

[4] Mäkelä J, Kiviniemi H, Laitinen S. Prevalence of perforated sigmoid diverticulitis is increasing. Dis Colon Rectum. 2002;45:955-61.

[5] Biondo S, Perea MT, Ragué JM, Parés D, Jaurrieta E. One-stage procedure in non-elective surgery for diverticular disease complications. Colorectal Dis. 2001;3:42-5. 
[6] American Gastroenterological Association. Recommendations from the American Gastroenterological Association on treatment and diagnosis. http://www.gastro.org/guidelines/acute-diverticulitis. Accessed 1 December 2018.

[7] Nagata N, Ishii N, Manabe N, Tomizawa K, Urita Y, Funabiki T, Fujimori S, Kaise M. Guidelines for Colonic Diverticular Bleeding and Colonic Diverticulitis: Japan Gastroenterological Association. Digestion. 2019;99 Suppl 1:1-26.

[8] Scheid V, Bensky D, Ellis A, Barolet R. Chinese herbal medicine: Formulas \& Strategies. 2nd ed. Seattle: Eastland Press; 2009: p. 685-772.

[9] Ogawa K, Nishijima K, Futagami F, Nakamura T, Nishimura G. Effectiveness of traditional Japanese herbal (Kampo) medicine, Daiobotanpito, in combination with antibiotic therapy in the treatment of acute diverticulitis: a preliminary study. Evid Based Complement Altern Med. 2013;2013:305414.

[10] Chan AW, Tetzlaff JM, Gotzsche PC, Altman DG, Mann H, Berlin JA, et al. SPIRIT 2013 explanation and elaboration: guidance for protocols of clinical trials. BMJ. 2013;346:e7586.

[11] Schechter S, Mulvey J, Eisenstat TE. Management of uncomplicated acute diverticulitis: results of a survey. Dis Colon Rectum. 1999;42:470-6.

[12] Kim DH, Yu KW, Bae EA, Park HJ, Choi JW. Metabolism of kalopanaxsaponin B and $\mathrm{H}$ by human intestinal bacteria and antidiabetic activity of their metabolites. Biol Pharm Bull. 1998;21:360-5.

[13] Fu PK, Yang CY, Tsai TH, Hsieh CL. Moutan cortex radicis improves lipopolysaccharide-induced acute lung injury in rats through anti-inflammation. Phytomedicine. 2012;19:1206-15.

[14] Oh GS, Pae HO, Choi BM, Jeong S, Oh H, Oh CS, et al. Inhibitory effects of the root cortex of Paeonia suffruticosa on interleukin-8 and macrophage chemoattractant protein-1 secretions in U937 cells. J Ethnopharmacol. 2003;84:85-9.

[15] Chung HS, Kang M, Cho $C$, et al. Inhibition of nitric oxide and tumor necrosis factor-alpha by moutan cortex in activated mouse peritoneal macrophages. Biol Pharm Bull. 2007;30:912-6.

\section{Tables}

Due to technical limitations, Table 1 is only available as a download in the supplemental files section.

\section{Figures}




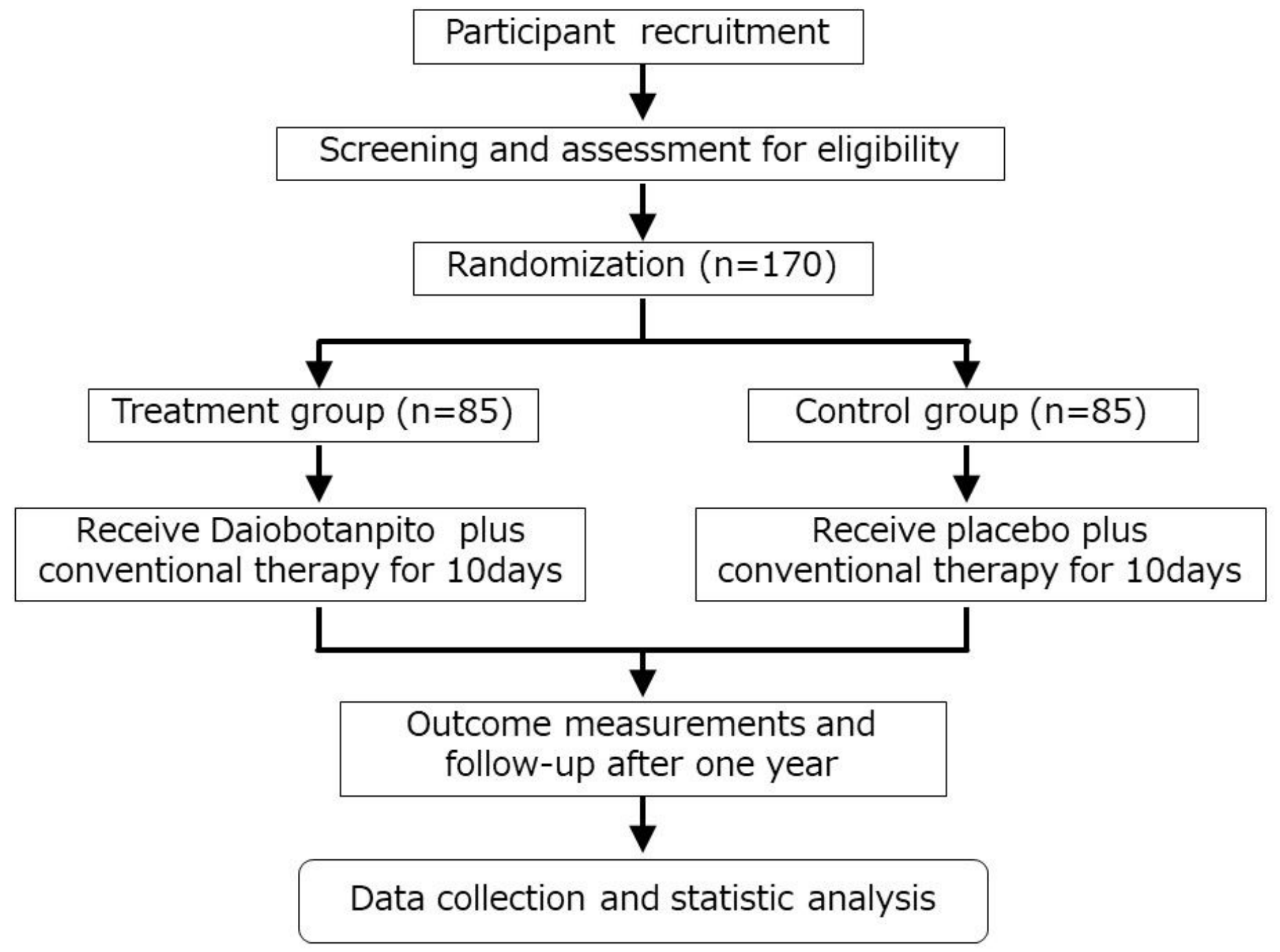

Figure 1

Study design flow chart

\section{Supplementary Files}

This is a list of supplementary files associated with this preprint. Click to download.

- 1903080gawaSPIRITFillablechecklist.doc

- Table1.pdf 